\title{
Effect of vibration on seepage feeding during low-pressure casting of ZL205A alloy
}

\author{
Ru-jia Wang, *Shi-ping Wu, and Wei Chen \\ School of Materials Science and Engineering, Harbin Institute of Technology, Harbin 150001, China
}

\begin{abstract}
Appropriate vibration can promote the feeding capacity of gravity casting, but the effect of vibration on the feeding of low-pressure casting is not clear. The effect of vibration on the seepage feeding of ZL205A alloy was investigated by vibration casting experiment and physical simulation. The aqueous solution of sodium carboxymethyl cellulose (CMC) with the same rheological characteristic of metal melt was used. The results show that vibration can improve the feeding capacity and reduce shrinkage defects of ZL205A alloy in lowpressure casting. The orthogonal physical simulation experiments indicate that vibration with low frequency and great exerted force can significantly improve the seepage velocity of non-Newtonian fluid with solid particles in porous medium. The seepage phenomenon in CMC solution shows that vibration can change the structure of accumulated particles at the seepage entrance, and thus open the blocked feeding channel. The numerical simulation of one-dimensional semisolid fluid seepage reveals that vibration can form a wave field in the porous medium, which can reduce the adhesion force between fluid and capillary wall and destroy the boundary layer of fluid, and thus promote the seepage velocity.
\end{abstract}

Key words: mechanical vibration; physical simulation; seepage; ZL205A alloy

CLC numbers: TG146.21 Document code:AＡrticle ID: 1672-6421(2019)01-040-06

Chrinkage porosity is one of the typical and most $\checkmark$ common defects in castings, which can dramatically affect the properties of the casting. It is very difficult to eliminate shrinkage porosity through subsequent thermomechanical treatments ${ }^{[1]}$ or forging process ${ }^{[2]}$, especially in the near-net shape casting process. Research has been done to prolong the service life of castings by reducing the shrinkage porosity ${ }^{[3-7]}$. It is found that shrinkage porosity in the casting is usually caused by obstruction of the feeding channel or insufficient feeding pressure of the alloy melt ${ }^{[8-11]}$. Low-pressure casting is an effective way to improve the feeding capacity and eliminate shrinkage defects in castings ${ }^{[12]}$. However, for the ZL205A alloy, which has been widely used in aviation, aerospace and military industries ${ }^{[13,14]}$, there are still many shrinkage porosities even if produced by lowpressure die casting ${ }^{[15]}$. Considerable studies indicate that vibration can improve the casting quality since Chernov first successfully refined grains of steel castings by gentle mould vibration ${ }^{[16,17]}$. Therefore, we creatively combine the vibration technology with low-pressure

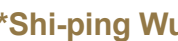

Male, Ph. D., Prof. Research interests: melt-treatment and metal forming technologies, solidification simulation.

E-mail: spwu@hit.edu.cn casting technology to improve the feeding capacity of ZL205A alloy castings. Mould vibration ${ }^{[16]}$ is one of the most convenient methods to improve the casting quality by vibration. Thus, mould vibration is introduced into the low-pressure casting process to reduce shrinkage porosity. For the high temperature of molten metal and the opacity of low-pressure casting process, physical simulation experiments are applied to explore the effect of vibration on the seepage feeding during the lowpressure casting of ZL205A alloy.

\section{Experimental procedure}

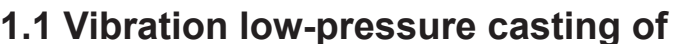

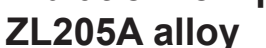

Vibration low-pressure casting is a new technology which combines the vibration process with lowpressure casting to improve feeding capacity. During the vibration low-pressure casting process, the metal melt fills the mould in low-pressure casting mode and then solidifies under vibration. As shown in Fig. 1, the vibration was transmitted to the mould through a \#45 steel rod, and formed a point vibration source which can concentrate the energy in the narrow feeding channel. The size of the casting is shown in Fig. 2. The pouring temperature of the melt was $700{ }^{\circ} \mathrm{C}$ and the holding pressure was $0.1 \mathrm{MPa}$. The vibration 


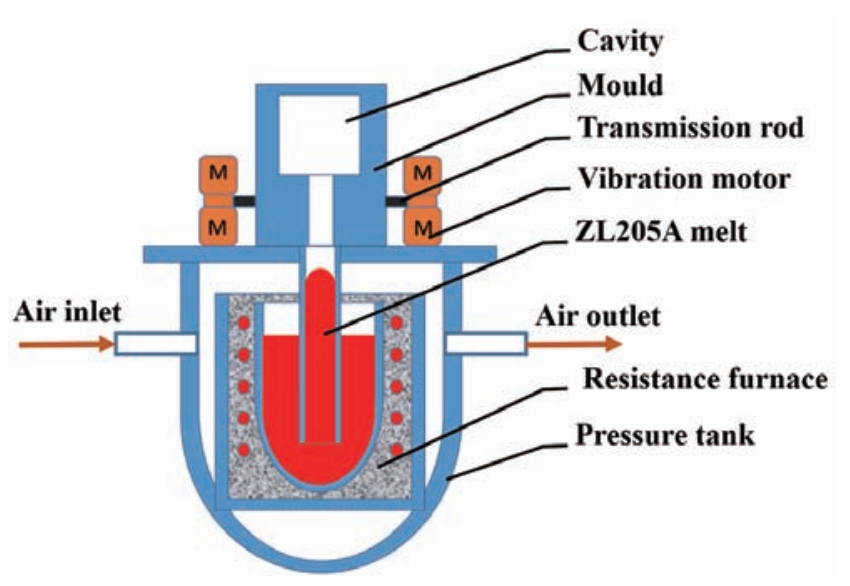

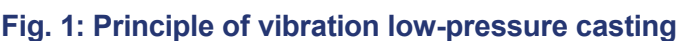

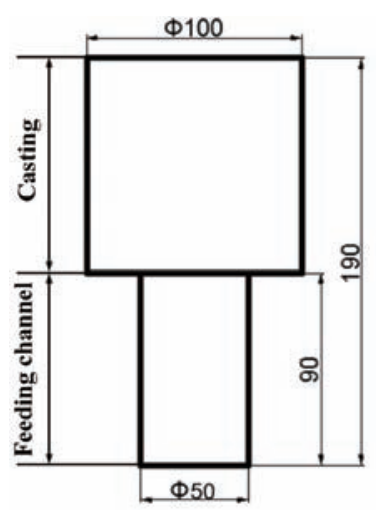

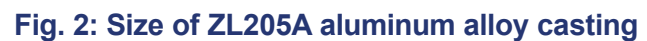

amplitude was $0.2 \mathrm{~mm}$, and the vibration frequency was 25 $\mathrm{Hz}$. The temperature at the start of vibration was $650{ }^{\circ} \mathrm{C}$. For comparison, the castings without vibration were also prepared under the same conditions.

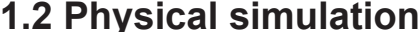

In order to study the effect of vibration on molten metal, the transparent $\mathrm{CMC}$ aqueous solution with the same rheological characteristic of the melt ${ }^{[18,19]}$ was selected as the experimental material, and acrylonitrile butadiene styrene resin (ABS) particles with similar density of CMC aqueous solution were added as the solidified equiaxed grains. The movement of ABS particles can also indicate the flow pattern of fluid under vibration. As shown in Fig. 3, the closely connected steel balls with a diameter of $2 \mathrm{~mm}$ were used to simulate the equiaxed grains of ZL205A alloy, and a mesh screen was used to prevent the drop of steel balls. The CMC aqueous solution with concentration of $4.5 \%$ and the steel balls were all packed in a cavity with thickness of $10 \mathrm{~mm}$. The front surface of the device was sealed with plexiglass, thus the fluid flow under vibration/non-vibration can be observed by digital industrial photogrammetry. Electromagnetic vibrators were set on both sides of the mould to vibrate the mould. The exerted force and frequency of vibration were regulated by an adjustable frequency converter. The fluid expelled from the top of the device under different processing parameters were weighed and converted into volume. The seepage velocity to estimate the seepage capacity was calculated through the seepage volume divided by seepage sectional area and seepage time. The physical simulation experiments were performed at $18{ }^{\circ} \mathrm{C}$. The orthogonal experimental scheme of three factors (holding pressure, frequency and exerted force) and three levels was designed, as shown in Table 1.

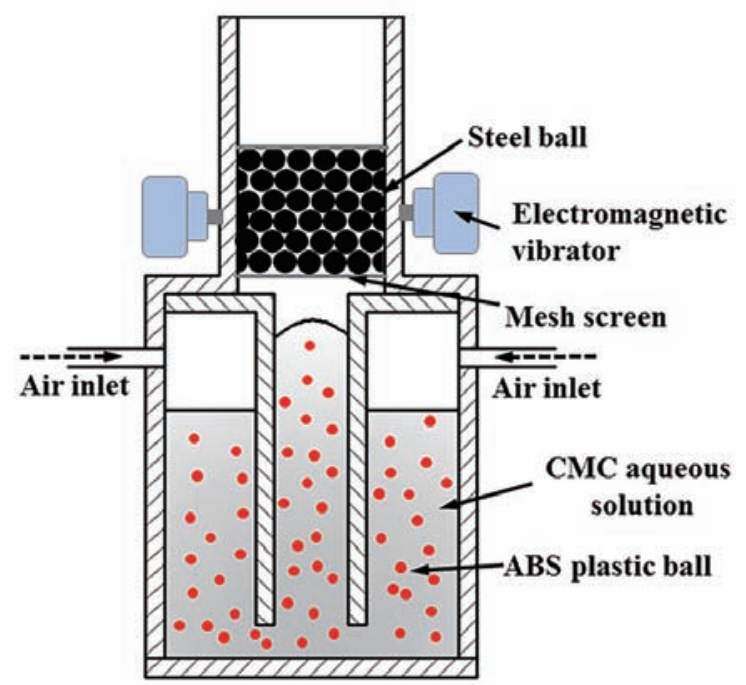

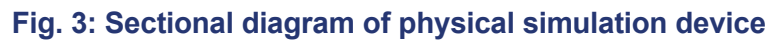

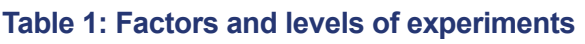

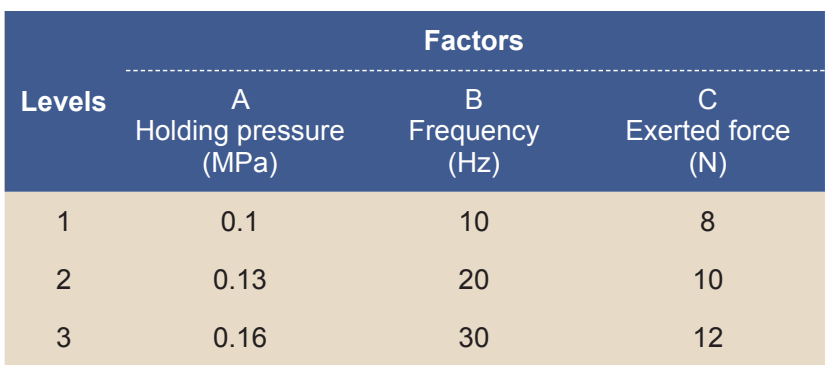

\section{Results and discussion}

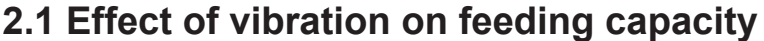

The castings were cut along the axis, and the area of shrinkage cavity in the castings was used to estimate the effect of vibration. As shown in Fig. 4, there is a large shrinkage cavity in the center of the casting solidified under non-vibration, while there is almost no shrinkage under vibration. Drainage method was used to measure the density of castings. The density of the casting without vibration is $2.7967 \mathrm{~g} \cdot \mathrm{cm}^{-3}$, lower than that of the casting with vibration at $2.8038 \mathrm{~g} \cdot \mathrm{cm}^{-3}$. The results indicate that vibration can improve the feeding capacity and reduce shrinkage defects in low-pressure casting.

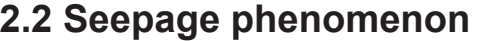

The dimensional size of ABS particles used in this experiment is between 40-60 mesh. Therefore, some of the particles can pass the gaps between the densely packed steel balls, and the remaining particles will accumulate at the seepage entrance and lead to higher resistance of seepage. The above seepage phenomenon is similar to that in the mushy solidified alloys (e.g. 

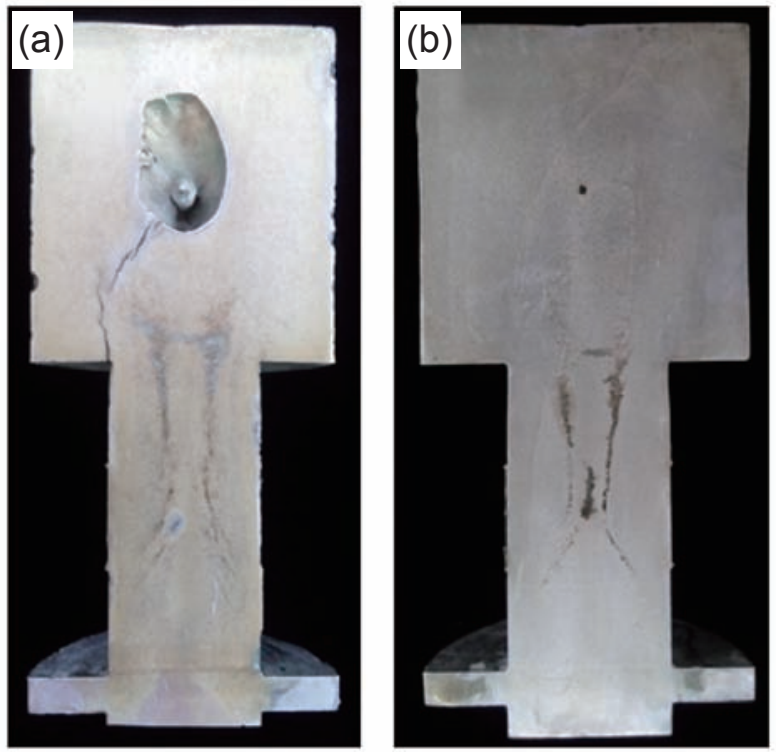

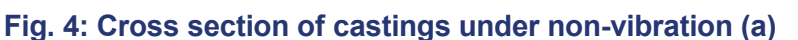

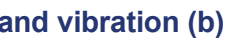

ZL205A alloy), in which the solidifying equiaxed grains easily accumulate at the feeding entrance and increase the feeding resistance. A tiny vibration on the ABS particles can change the structure of the accumulated particles and reduce the resistance of semisolid seepage.

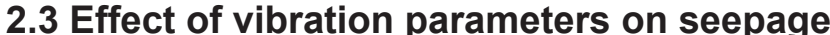

As shown in Fig. 5, the seepage velocity of a semisolid fluid in a porous medium increases with the increment of holding pressure under non-vibration. Appropriate vibration can greatly improve the seepage velocity (e.g., when the frequency is $30 \mathrm{~Hz}$, the exerted force is $10 \mathrm{~N}$ and the holding pressure is $0.16 \mathrm{MPa}$ ), whereas it will decrease under inappropriate vibration. The effects of vibration on the seepage are different under different holding pressures. The seepage velocity under the proper vibration $(10 \mathrm{~Hz}, 10 \mathrm{~N})$ is twice that under non-vibration when the holding pressure is intermediate value, $0.13 \mathrm{MPa}$. However, vibration has little influence on the seepage velocity when the holding pressure is of relatively great value, $0.16 \mathrm{MPa}$.

Table 2 shows the results of the orthogonal test. It can be concluded from the analysis of range $(R)$ in Table 2 that holding

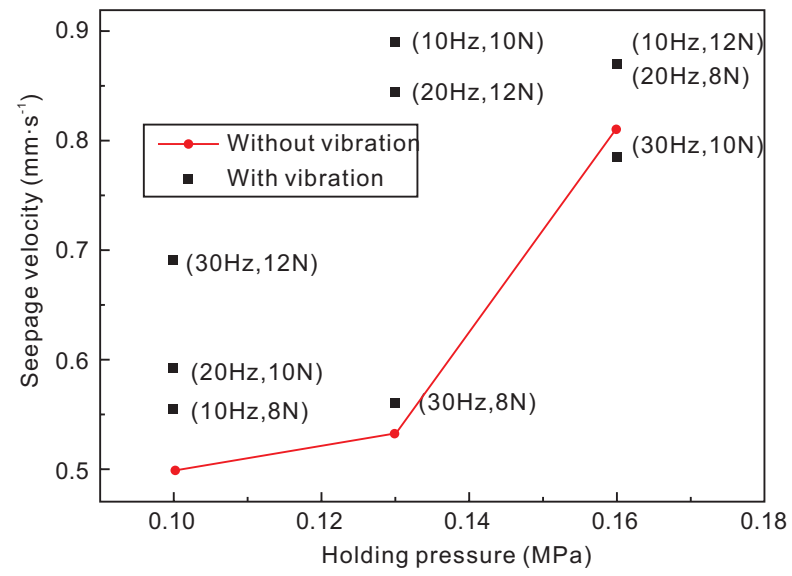

Fig. 5: Effect of vibration on seepage flow pressure influences seepage velocity the most, exerted force influences less and frequency influences the least. The optimized parameters to obtain the seepage velocity are the greatest holding pressure and the vibration with great exerted force and low frequency. As shown in Fig. 6, the seepage velocity increases with the increment of holding pressure and exerted force but decreases with the increase of vibration frequency. This is because the pressure gradient increases with the increase of holding pressure when the pressure of the seepage outlet is equal to that of the atmosphere. According to Darcy's law, the seepage velocity becomes faster with a higher pressure gradient. However, in the practical low-pressure casting process, the seepage velocity cannot increase any more even if the holding pressure continually increases once the feeding channel is blocked. Therefore, the unimpeded feeding channel is a prerequisite condition to improve seepage velocity by increasing holding pressure. In terms of the above seepage phenomenon in the semisolid fluid under vibration, the vibration can change the structure of accumulated particles. The greater the exerted force is, the more easily the blocked structure is destroyed, and consequently, the seepage velocity becomes faster.

On the other hand, according to the equation of vibration acceleration of particle:

$$
a=4 \pi^{2} A f^{2}
$$

where $a$ is the vibration acceleration of particle, $A$ is the vibration amplitude and $f$ is the vibration frequency. When the exerted force is constant, the amplitude becomes smaller with higher frequency, and therefore, the accumulated structure changes very little, and consequently, the seepage velocity becomes slower. Meanwhile, mechanical vibration propagates in the castings in the form of wave, and its amplitude decreases with the increse of the propagation distance. The higher the vibration frequency is, the more serious the attenuation of the mechanical vibration. Therefore, the low-frequency mechanical vibration is more suitable for decreasing the shrinkage porosity during low-pressure casting compared with the high-frequency mechanical vibration.

\subsection{Calculation of seepage flow in porous}

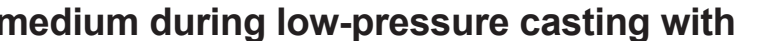

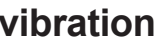

The pressure equation of a semisolid fluid in a one-dimensional porous medium under vibration and low-pressure is obtained by coupling the continuity equation with the momentum equation based on the assumption that solid particles are incompressible, the semisolid fluid is weakly compressible, the viscosity of the fluid is constant, and the seepage velocity and seepage pressure gradient satisfy Darcy's law ${ }^{[20]}$.

$$
\left.\begin{array}{l}
\frac{\partial^{2} p}{\partial x^{2}}=\frac{1}{\eta} \frac{\partial p}{\partial t} \\
p(0, t)=p_{w}+p_{0} \sin \omega t \\
p(L, t)=p_{e} \\
p(x, 0)=p_{w}+\frac{p_{e}-p_{w}}{L} x
\end{array}\right\}
$$

where $p_{w}$ is the stable pressure at the entrance of seepage, $\mathrm{Pa}$; 


\begin{tabular}{|c|c|c|c|c|}
\hline \multirow{2}{*}{ No. } & \multicolumn{3}{|c|}{ 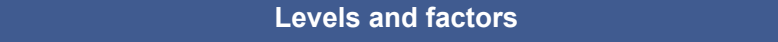 } & \multirow{2}{*}{ Seepage velocity $\left(\mathrm{mm} \cdot \mathrm{s}^{-1}\right)$} \\
\hline & $\mathrm{A}(\mathrm{MPa})$ & $\mathrm{B}(\mathrm{Hz})$ & $\mathrm{C}(\mathrm{N})$ & \\
\hline 1 & 1 & 1 & 1 & 0.555 \\
\hline 2 & 1 & 2 & 2 & 0.592 \\
\hline 3 & 1 & 3 & 3 & 0.692 \\
\hline 4 & 2 & 1 & 2 & 0.890 \\
\hline 5 & 2 & 2 & 3 & 0.845 \\
\hline 6 & 2 & 3 & 1 & 0.560 \\
\hline 7 & 3 & 1 & 3 & 0.872 \\
\hline 8 & 3 & 2 & 1 & 0.868 \\
\hline 9 & 3 & 3 & 2 & 0.785 \\
\hline$K_{1}$ & 1.838 & 2.317 & 1.985 & \\
\hline$K_{2}$ & 2.295 & 2.307 & 2.268 & \\
\hline$K_{3}$ & 2.527 & 2.037 & 2.407 & \\
\hline$k_{1}$ & 0.613 & 0.772 & 0.662 & \\
\hline$k_{2}$ & 0.765 & 0.768 & 0.757 & \\
\hline$k_{3}$ & 0.842 & 0.678 & 0.802 & \\
\hline$R$ & 0.230 & 0.093 & 0.142 & \\
\hline $\begin{array}{l}\text { Sequence of } \\
\text { factors }\end{array}$ & & & & \\
\hline $\begin{array}{l}\text { Optimization } \\
\text { level }\end{array}$ & A3 & B1 & C3 & \\
\hline $\begin{array}{c}\text { Excellent } \\
\text { combination }\end{array}$ & & & & \\
\hline
\end{tabular}

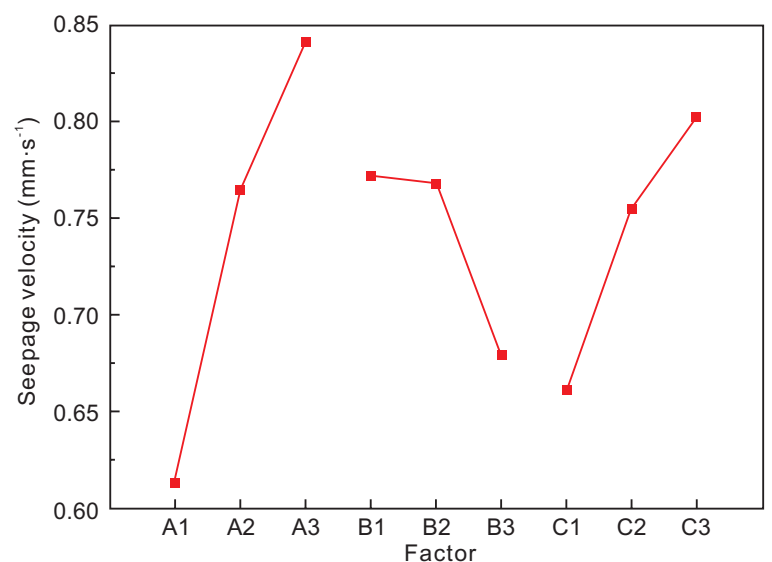

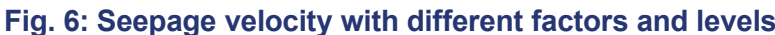

$p_{0}$ is the additional pressure of vibration, $\mathrm{Pa} ; p_{e}$ is the stable pressure at the outlet of seepage, $\mathrm{Pa}$; $L$ is the length of porous medium, $\mathrm{m} ; \omega$ is the angular frequency of vibration, $\operatorname{rad} \cdot \mathrm{s}^{-1}$; and $\eta$ is the pressure transmitting coefficient, $\mathrm{m}^{2} \cdot \mathrm{s}^{-1}$. And $\eta$ can be expressed by the following equation:

$$
\eta=\frac{K}{\phi \mu C}
$$

where $K$ is the permeability of porous medium, $\mathrm{m}^{2} ; \phi$ is the porosity ratio of porous medium; $\mu$ is the viscosity of fluid, $\mathrm{Pa} \cdot \mathrm{s} ; C$ is the comprehensive compression coefficient which can be calculated by the sum of compressibility of fluid and porous medium, $\mathrm{Pa}^{-1}$.

Under the vibration, the additional pressure satisfies the following equation:

$$
p_{0}=\frac{F}{S}
$$

where $F$ is exerted force of vibration, N; and $S$ is the action area of exerted force, $\mathrm{m}^{2}$.

In this study, the length of porous medium increases continually due to the accumulation of solid particles in the semisolid liquid during the seepage progress. According to the volume conservation of solid particles, the relationship between the volume of seepage discharge and the length of porous medium can obtained by the following equation,

$$
\frac{1}{6} \pi d^{3} \rho_{n} \int_{0}^{t} q d t=S \Delta L(1-\phi)
$$


where $q$ is the seepage discharge, $\mathrm{m}^{3} \cdot \mathrm{s}^{-1} ; d$ is the equivalent diameter of solid particles, $\mathrm{m} ; \rho_{n}$ is the particle concentration of semisolid fluid, $\mathrm{m}^{-3}$; and $\Delta L$ is the increment of length of porous medium due to the accumulation effect of particles, $\mathrm{m}$.

The finite difference method was used to solve Eq. (2) and (5). The calculation parameters are shown in Table 3.

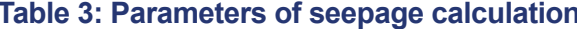

\begin{tabular}{ccccccccccccc}
\hline Value & 0.2 & 0.02 & 0.1 & 50 & 2 & $4.76 \times 10^{-10}$ & $9.3 \times 10^{-5}$ & 0.264 & $1.28 \times 10^{5}$ & $20 \pi$
\end{tabular}

Figure 7 shows the distribution and evolution of seepage pressure in the porous medium by solving Eqs. (1) and (4) numerically. As shown in Fig. 7, the pressure in the seepage process is changing like a wave under vibration, and the changing pressure can make the flow velocity of fluid in capillary channels of porous medium change periodically, which means vibration forms an elastic wave field in a porous medium. The pressure transmitting coefficient Eq. (1) is calculated as $5.6 \mathrm{~m}^{2} \cdot \mathrm{s}^{-1}$. Thus, the seepage pressure can rapidly achieve an equilibrium state, which results in the linear pressure gradient in the porous medium. Therefore, the pressure gradient along the porous medium changes in sinusoidal since the pressure of vibration source is sinusoidal. The vibration of the fluid in the boundary layer formed because the adhesion force between the semisolid liquid and the capillary wall can reduce the adhesion force, so that the elastic wave field can significantly reduce the influence of capillary force on seepage flow and increase the movement of fluid through the porous medium ${ }^{[20]}$. Meanwhile, mechanical vibration can also destroy the boundary layer ${ }^{[21]}$. Consequently, the effective size of the capillary can be increased.

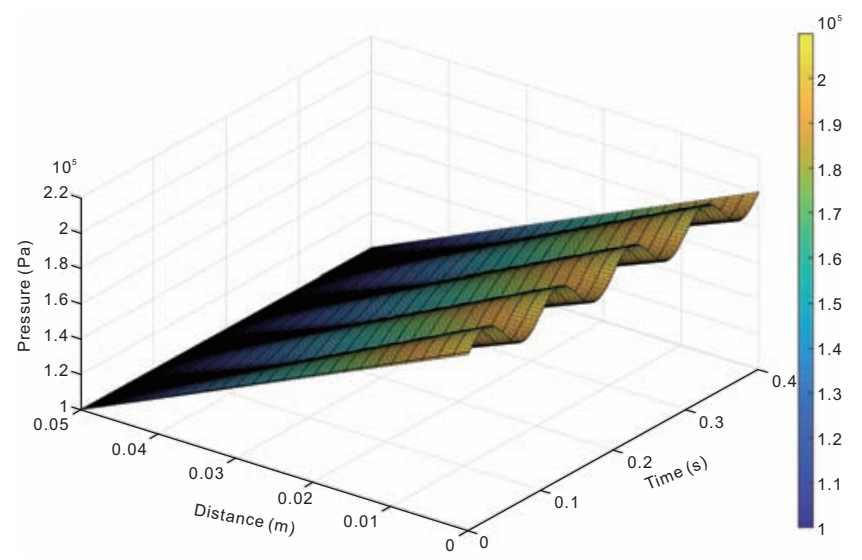

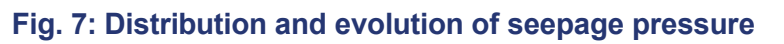

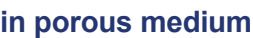

In addition, the $4.5 \% \mathrm{CMC}$ aqueous solution has viscoelastic rheological characteristics, and the solution can only flow when the pressure gradient of the porous medium is greater than the critical pressure. The critical pressure of fluid decreases with increasing the size of the capillary under the same pressure gradient ${ }^{[18]}$. The fluid in some small capillaries in the porous medium cannot flow under the pressure which is smaller than the critical pressure, as shown in Fig. 8(a). Part of the capillary which cannot flow starts to move when the

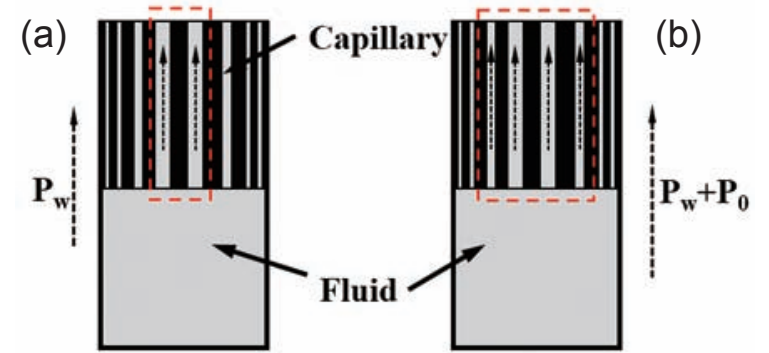

Fig. 8: Effect of vibration on capillary flow in porous $\square \square \square \square \square$

pressure is greater than the critical pressure due to the additional pressure of vibration. Consequently, the permeability of the porous medium increases, as shown in Fig. 8(b).

The propagation of vibration could produce tensile and shear stress in the melt ${ }^{[22]}$. The apparent viscosity of the melt reduces with increasing of shear rate ${ }^{[23]}$, as shown in Eq. (6) ${ }^{[24]}$.

$$
\frac{\eta-\eta_{\infty}}{\eta_{0}-\eta_{\infty}}=\frac{1}{1+k \dot{\gamma}}
$$

where $\eta$ is the melt viscosity; $\eta_{0}$ and $\eta_{\infty}$ are the asymptotic viscosity values at zero and infinite shear rates, respectively; $k$ is the parameters related to time; and $\dot{\gamma}$ is the shear strain velocity.

In addition, the shear stress caused by vibration will consume the vibration energy in the form of internal friction. Therefore, the local temperature of the fluid will increase. According to theory ${ }^{[25]}$, the viscosity of the fluid decreases with the increase of temperature. Hence, apparent viscosity of the melt can also be reduced by thermal effect of vibration, which will promote the flow velocity of fluid. The thermal effect and the shear rate become greater with the increase of the amplitude and frequency, thus, vibration can reduce the viscosity of the fluid more obviously according to Eq. (6). However, the velocity and direction of the fluid particle are constantly changing under vibration, which makes the fluid particle move from one layer to another and produces additional resistance to the flow of the fluid. The turbulence degree of fluid and the additional resistance caused by turbulence will increase by increasing the vibration amplitude and frequency. Vibration will reduce the seepage velocity when the obstructing effect is greater than the promoting effect.

\section{Conclusions}

(1) Vibration can improve the feeding capacity and reduce 
shrinkage defects of ZL205A alloy in low-pressure casting.

(2) Vibration can change the structure of accumulated particles at the seepage entrance, thus open the blocked feeding channel. Meanwhile, vibration can form a wave field in the porous medium, which can reduce the adhesion force between fluid and the capillary wall and destroy the boundary layer of fluid, and thus promote the seepage velocity.

(3) Holding pressure influences seepage velocity the most, exerted force influences less and frequency influences the least. The vibration with low frequency and great exerted force (e.g., when the frequency is $10 \mathrm{~Hz}$, the exerted force is $12 \mathrm{~N}$ and the holding pressure is $0.16 \mathrm{MPa}$ ) can significantly improve the seepage feeding of semi-solid fluid in porous medium, however, if the obstructing effect of turbulence induced by vibration is greater than the promoting effect, the vibration will reduce the seepage velocity.

\section{References}

[1] Sang Baoguang. Numerical simulation and experimental study of inclusions and macrosegregation in the bottom-filled heavy steel Ingots. PhD Thesis, Institute of Metal Research, Chinese Academy of Sciences, China, 2010.

[2] Wang Jiaqi, Fu Paixian, Liu Hongwei, et al. Shrinkage porosity criteria and optimized design of a 100-ton 30Cr2Ni4MoV forging ingot. Materials and Design, 2012, 35: 446-456.

[3] Hardin R, Beckermann C. Prediction of the Fatigue Life of Cast Steel Containing Shrinkage Porosity. Metallurgical \& Materials Transactions A, 2009, 40(3):581.

[4] Li Bo, Shen Yifu, Hu Weiye. Casting defects induced fatigue damage in aircraft frames of ZL205A aluminum alloy - A failure analysis. Materials \& Design, 2011, 32(5):2570-2582.

[5] Buffière J Y, Savelli S, Jouneau P H, et al. Experimental study of porosity and its relation to fatigue mechanisms of model Al$\mathrm{Si} 7-\mathrm{Mg} 0.3$ cast Al alloys. Materials Science and Engineering: A, 2001, 316 (1-2): 115-126.

[6] Lados D A, Apelian D. Fatigue crack growth characteristics in cast Al-Si-Mg alloys: part I. Effects of processing conditions and microstructure. Materials Science and Engineering: A, 2004, 385: 200-211.

[7] Linder J, Axelsson M, Nilsson $\mathrm{H}$. The influence of porosity on the fatigue life for sand and permanent mould cast aluminium. International Journal of Fatigue, 2006, 28 (12): 1752-1758.

[8] Wang Rujia, Wu Shiping, Chen Wei. Mechanism of burst feeding in ZL205A casting under mechanical vibration and low pressure[J]. Transactions of Nonferrous Metals Society of China, 2018, 28(8): 1514-1520.

[9] Mitrasinovic A, Hernández F C R, Djurdjevic M , et al. On-line prediction of the melt hydrogen and casting porosity level in 319 aluminum alloy using thermal analysis. Materials Science \& Engineering A, 2006, 428(1): 41-46.
[10] Sun Y, Luo Jian, Mi G F, et al. Numerical simulation and defect elimination in the casting of truck rear axle using a nodular cast iron. Materials \& Design, 2011, 32(3):1623-1629.

[11] Verran G O, Rui P K M, Rossi M A. Influence of injection parameters on defects formation in die casting Al12Si13Cu alloy: Experimental results and numeric simulation. Journal of Materials Processing Technology, 2006, 179(1-3):190-195.

[12] Zhu L, Wang D, Zhang H. The Research and Application of Feeding Mechanism in the Solidification Process of Foundry Alloy. Special Casting \& Nonferrous Alloys, 1997, 6:34-38. (In Chinese)

[13] Zhang Ming, Zhang Weiwen, Zhao Haidong, et al. Effect of pressure on microstructures and mechanical properties of AlCu-based alloy prepared by squeeze casting. Transactions of Nonferrous Metals Society of China, 2007, 17(3): 496-501.

[14] Li Min, Wang Hongwei, Wei Zunjie, et al. The effect of $Y$ on the hot-tearing resistance of Al-5wt.\% Cu based alloy. Materials \& Design, 2010, 31(5): 2483-2487.

[15] Wang Y, Shi-Ping Wu, Xue X, et al. Formation mechanism and criterion of linear segregation in ZL205A alloy. Transactions of Nonferrous Metals Society of China, 2014, 24(11):3632-3638.

[16] Maltais A, Fiset M, Dubé D. An apparatus to grain refine metals and alloys with mold vibrations. Review of Scientific Instruments, 2005, 76(10): 647-129.

[17] Wu Shi-ping, Wang Ru-jia, Wang Ye, et al. Reduction of shrinkage porosities in aluminum alloy castings by external pressure fluctuation under gravity field]. China Foundry, 2018, 15(5): 372-377.

[18] Li Defu. Rheological research on flow-type defects in castings. Harbin Institute of Technology, 1995, Harbin.

[19] Wang Rujia, Wu Shiping, Chen Wei. Unified wave equation and numerical simulation of mechanical wave propagation in alloy solidification. Simulation, 2019, 95(1): 3-10.

[20] Zhi S Y, Xiang W A, Hua L J. Vibration Drag-reduction of Pipeline Transport of High Density Slurry. Mining \& Metallurgical Engineering, 2001, 21(4): 4-6. (In Chinese)s

[21] Beresnev I A, Johnson P A. Elastic-wave stimulation of oil production: A review of methods and results. Geophysics, 1994, 59(6): 1000-1017.

[22] Guan R G, Wang X, Shang Y Q, et al. Shear Model of Metal Melt Flowing on Vibration Wall and Effect of Shear Stress on Solidification Microstructure. Acta Metallurgica Sinica (English Letters), 2018: 1-9.

[23] Kattamis T Z, Piccone T J. Rheology of semisolid Al-4.5\% Cu1.5\% Mg alloy[J]. Materials Science and Engineering: A, 1991, 131(2): 265-272.

[24] Roberts G P, Barnes HA, Carew P. Modelling the flow behaviour of very shear-thinning liquids. Chemical Engineering Science, 2001, 56(19):5617-5623.

[25] Andrade E N C. LVIII. A theory of the viscosity of liquids - Part II. The London, Edinburgh, and Dublin Philosophical Magazine and Journal of Science, 1934, 17(113): 698-732. 